\title{
Christian Braun. 2017. Das Althochdeutsche aus textlinguistischer Sicht. Soziopragmatische Einordnung und ausgewählte textgrammatische Studien (Lingua Historica Germanica 14). Berlin, Boston: De Gruyter. xii, 380 S.
}

Besprochen von Eckhard Meineke: Friedrich-Schiller-Universität Jena, Institut für Germanistische Sprachwissenschaft, Fürstengraben 30, D-07737 Jena, E-Mail: Eckhard.Meineke@uni-jena.de

https://doi.org/10.1515/zrs-2018-0003

„In den Jahren der Auseinandersetzung mit der hier vorliegenden Studie habe ich mich das eine oder andere Mal gefragt, was ich mir nur dabei gedacht habe, einen textlinguistischen Zugriff auf die althochdeutsche Sprachepoche zu wagen“ (S. v).

Mit dieser captatio benevolentiae beginnt das Buch des Grazer Sprachwissenschaftlers. Denn für jeden der beiden Bereiche liege „doch recht viel“ an Sekundärliteratur vor, so dass allein das Vorhaben, sich in das Althochdeutsche und die Textlinguistik umfassend einzulesen, nie zufriedenstellend umgesetzt sein könne. Aber die Antwort auf die Frage sei stets die gleiche geblieben: Interesse am Thema. Auch der Leser fragt sich, was ihn an der Thematik interessieren kann, ob er sich überhaupt für sie interessieren muss, und wenn ja, ob das Buch überzeugt und neue Erkenntnisse enthält.

Braun stellt in den ersten beiden inhaltlichen Kapiteln die theoretischen Grundlagen der Textlinguistik vor (S. 6-93). Kapitel 2 gilt dem Text als Gegenstand linguistischer Betrachtung. Behandelt werden Rhetorik und Stilistik als Vorläufer der Textlinguistik, die Zugriffsweisen der Textlinguistik (strukturalistisch, strukturalistisch-funktional, semantisch, pragmatisch-kommunikativ, kognitiv) bis hin zu den integrativen Ansätzen von Brinker \& Ausborn-Brinker (2010) und de Beaugrande \& Dressler (1981), die so neu nicht sind. Das dritte Kapitel befasst sich mit theoretischen Grundlagen: Verortung des Textbegriffs, Texte in Textklassen, Texttypen, Textmuster, Textsorten, sodann Texte in Netzen (Text und Diskurs, Textallianz, Textensemble, Hypertext). Dann werden die Eigenschaften von Texten behandelt: 1 . externe Merkmale, 2. textinterne und textinduzierte Eigenschaften. Diese gliedern sich in a. Kohärenz und Kohäsion, b. Merkmale textgrammatischer Kohärenz (Wiederaufnahme/Rekurrenz, Konnexion, grammatische Kategorien des Verbs, Textoberflächengestaltung/Textarchitektonik), c. Textthema und d. Textfunktion.

In Teil 1 von Kapitel 4 „Das Althochdeutsche aus textlinguistischer Sicht“ stellt Braun im ersten Punkt „Allgemeine Überlegungen“ (S. 94-124) das „Althochdeutsche als Periode in der deutschen Sprachgeschichte“ vor. Der zweite Teil des Kapitels enthält Überlegungen zur „Verbindung von historischer Soziopragmatik und historischer Textlinguistik innerhalb eines integrativen Mo- 
dells“ (vgl. S. 124-138). Ab S. 124 also scheint das Buch interessant zu werden, jedenfalls aus der Sicht eines Fachkollegen mit längerer Erfahrung und sich daraus ergebendem Ennui an manchem, was neuere Forschung ,wiederentdeckt“ oder zumindest anders und komplizierter formuliert hat als die frühere.

Es ist sicherlich eine angemessene Verfahrensweise - aber besser vor dem Schreiben der Arbeit, nicht als deren Teil -, sich die Gegenstände Textlinguistik und Althochdeutsch klarzumachen, bevor man den Versuch einer textlinguistischen Aufarbeitung althochdeutscher Sprachdenkmäler unternimmt. Das auch deshalb, weil unlängst ein Buch zu den althochdeutschen Glossen erschien, das auf der Basis zweier aus dem Kontext gerissener Zitate aus de Beaugrande \& Dressler und Brinker \& Ausborn-Brinker einen Textbegriff vertritt, aufgrund dessen sich althochdeutsche Glossen als Texte verstehen lassen sollen (vgl. Schiegg 2015). Es spricht für Braun, dass er diese Science Fiction und ihre „Traditionslinie“ auf einer Seite abtut, ebenso wie die „Textsorten“klassifikation von Alexander Schwarz (2000), der als Textsorten des Althochdeutschen Schule, Gottesdienst, Lebenspraxis, Erbauung, antiquarisches Interesse, Verwaltung, Politik und Traditionsbildung ansetzt (Braun, S. 122f.). Braun schreibt (S. 123) anhand des Hildebrandsliedes höflich, dass Schwarz wohl auf dem richtigen Weg sei und eine Gliederung der Textsorten nach Kommunikationsbereichen - denn diese verwechselt Schwarz mit Textsorten - als „durchaus Erkenntnis fördernd“ erscheine - was man von jedem Fehler sagen kann, aus dem man lernen kann. Dann aber führt er selbst unüberlegte Klassifizierungen auf, indem er als Kommunikationsbereich des Hildebrandsliedes „antiquarisches Interesse“ nennt und als Textsorte „Heldenlied“. Braun berücksichtigt das Buch „Textgebundenheit“ von Schützeichel (1981) nicht, in dem die Anwendung des Begriffs Heldenlied auf das Hildebrandslied diskutiert wird, das alles andere als ein Heldenlied ist. Und es geht auch nicht um antiquarisches Interesse.

Nach 124 Seiten, einem Drittel des Buches, mit dem sich der Verfasser seinen Gegenstand erarbeitet hat, dessen Inhalt aber unter Fachleuten bekannt ist, kommt Braun zu seinem eigentlichen Thema. Hierzu soll zunächst die „Verbindung von historischer Soziopragmatik und historischer Textlinguistik innerhalb eines integrativen Modells“ (S. 124) geleistet werden. Braun stellt eine Liste von Forderungen zusammen, die 1. allgemein an eine „postsystemlinguistische“ Sprachwissenschaft und 2. an die historische Linguistik gerichtet werden. Als Quintessenz formuliert er, dass der Linguist zusätzlich noch zu sein habe: Historiker, Soziologe, Psychologe, Diskurswissenschaftler, Medienwissenschaftler, Kulturwissenschaftler, Handlungstheoretiker, Philosoph, ..., also ein universaler Gelehrter. Und dieses Wissen stelle nur die Grundlage für die eigentliche systemimmanente Sprachanalyse dar. „Das ist Hybris.“(S. 129). 
Der universale Anspruch wird aber nicht eingelöst. Denn die Kriterien, nach denen Braun die Textdenkmäler des Althochdeutschen schließlich beschreibt (vgl. S. 145-247), entspringen bescheideneren Ansprüchen: 1. Auftraggeber/Verfasser/Übersetzer/Schreiber als Ausführende, 2. Adressat/Rezipient/das intendierte Publikum, 3. Zeitrahmen, 4. Schreibort, 5. Kommunikationsbereich, 6. diskursive Verortung, 7. Art und Weise der Aufzeichnung, 8. Motivation der Niederschrift/das Verfasserinteresse/der Grund der Aufzeichnung (vgl. S. 141).

Damit wird es kritisch. Denn diese Beschreibung, ein Viertel der Arbeit, mit viel Leerraum zwischen den jeweils acht Abschnitten, beruht auf dem Ausschreiben bereits vorhandener Informationen über die althochdeutschen Textdenkmäler. Zusammen mit den Kapiteln über Textlinguistik und das Althochdeutsche stellen fast $60 \%$ der Arbeit ein Referat vorgängiger Forschung dar, aber keine eigene wissenschaftliche Leistung. Und was diese Beschreibung mit Textlinguistik zu tun haben soll, erschließt sich dem Rezensenten auch nicht. Denn was erfährt man, gar Neues? Das sei hier am Beispiel der Murbacher Hymnen gezeigt. 1. Auftraggeber/Verfasser/Übersetzer/Schreiber als Ausführende: „Die Murbacher Hymnen sind von Klerikern für Kleriker zum Zwecke schulischer Vertiefung interlinear in den lateinischen Text eingefügt worden.“ 2. Adressat/Publikum: „Als Adressat ist der sich mit den Hymnen im schulischen Kontext auseinandersetzende Kleriker anzusehen.“ 3. Chronologie: „Die Niederschrift der Murbacher Hymnen erfolgte wohl noch im ersten Viertel des 9. Jahrhunderts, beide Teile in geringem zeitlichem Abstand nacheinander.“ 4. Schreibort: „Als Orte der Niederschrift gelten die Reichenau und Murbach.“ 5. Kommunikationsbereich: „Der Text kann dem Kommunikationsbereich Klösterliches Schulwesen zugeordnet werden.“ 6. Diskursive Verortung: „Als Interlinearversion lassen sich die Murbacher Hymnen im Grunde nicht nur an den lateinischen Primärdiskurs anbinden, vielmehr sind sie als nicht eigenständiger Text Bestandteil desselben.“ 7. Art und Weise der Aufzeichnung: Kurzreferat der Handschriftenbeschreibung von Lothar Voetz in Bergmann (2013). 8. Motivation der Niederschrift/Verfasserinteresse/Grund der Aufzeichnung: Braun folgt hier Nikolaus Henkel: die interlineare Niederschrift der Murbacher Hymnen diene ,allein dem Ziel der tieferen morphosyntaktischen Erschließung des lateinischen Textes“. Verwendungszweck sei der Gebrauch im Rahmen der klösterlichen Ausbildung. „Eine dichterische Eigenständigkeit der Volkssprache bzw. eine solche als volkssprachiger Text generell wird demzufolge nicht angenommen“ (S. 197f.).

In dieser Weise werden alle Texte beschrieben. Der althochdeutsche Tatian etwa wird dem Kommunikationsbereich „Sonstiges“ zugeordnet (S. 228). Das erscheint nichtssagend. Wer umfassend über die althochdeutschen Texte informiert werden will, greift zum Sammelwerk von Bergmann (2013). 
Die Beobachtungen zu den acht Kriterien werden sodann in Kapitel 6 „Parametergestützte Auswertungen und Überlegungen“ (S. 248-277) ausgewertet. Hier erfährt man, dass das althochdeutsche Schrifttum zu seinem überwiegenden Anteil als klerikal-monastische, d.h. elitäre Gruppensprache aufgefasst werde (S. 276).

Das achte Kapitel „Ausgewählte textgrammatische Studien althochdeutscher Texte“ (S. 278-348) - gemeint ist sicher „Studien zu althochdeutschen Texten“ -, nach Abzug des Raums für die Texte und Faksimiles 15\% der Studie, wäre damit der Teil der Arbeit, der Eigenes bieten könnte. Als Materialgrundlage kämen, so Braun, nur jene Sprachdenkmäler in Frage, die auch tatsächlich Texte seien, d.h. ausschließlich jene, deren Zielsprache auch wirklich das Althochdeutsche sei und die als Rezipienten die Laiengemeinde als illitterati adressierten (vgl. S. 279). Behandelt werden 1. das Fränkische Taufgelöbnis, 2. das St. Galler Credo, 3. die Fuldaer Beichte, 4. die Lorscher Beichte, 5. die Exhortatio ad plebem christianam, 6. Otlohs Gebet, 7. das Augsburger Gebet, 8. das Petruslied sowie 9. „Spezialfälle: Die Freisinger Gebete/so genannten Gebete Sigiharts“. Zunächst wird der Text zitiert. Dann werden dargestellt: 1. Rekurrenz, 2. Konnektoren, 3. Verben/Prädikate, 4. Thema/Thematische Entfaltung. Es folgen allgemeine Bemerkungen. Auch hier fragt man sich, welchen Erkenntniswert es hat, wenn etwa bei der Lorscher Beichte unter 2. die drei Konnektoren inti, inther („mit Artikel verschmolzen“) und thanne aufgeführt werden (S. 308). Was es soll, wenn unter 3. „Verben/Prädikate“ die Verben der 16 Sätze mit grammatischer Bestimmung aufgelistet werden, also etwa (S. 309): „Satz 1: gihu (1. Person, Singular, Präsens, Indikativ, aktiv); Zweitstellung, Subjekt ih“ (es folgen analog die übrigen 15 Verben). Und wo ist der Sinn der „textlinguistischen“ Beschreibung, wenn es unter „4. Thema/Thematische Entfaltung“ heißt, der Beichtende bekenne, etwas Bestimmtes nicht in der Art und Weise getan zu haben, wie er sollte bzw. wie es seine Pflicht gewesen wäre (S. 309)? Wo liegt bei der Textsorte Beichte der Erkenntniswert der ,allgemeinen Bemerkung“ (S. 310), dass das Pronomen ih „höchstfrequent“ (55x) auftritt?

Fazit ist: Brauns Buch besteht ganz überwiegend aus Forschungsreferaten, im Fall des Althochdeutschen und der Übersicht über die Sprachdenkmäler aber weit unter der Detailauflösung der vorgängigen Veröffentlichungen. Der Beitrag der eigenen „textlinguistischen Erforschung“ von neun kleinen Texten ist auf vordergründige Strukturbeschreibungen beschränkt. 


\section{Literatur}

Beaugrande, Robert-Alain de \& Wolfgang U. Dressler. 1981. Einführung in die Textlinguistik (Konzepte der Sprach- und Literaturwissenschaft 28). Tübingen: Max Niemeyer.

Bergmann, Rolf (Hg.). 2013. Althochdeutsche und altsächsische Literatur. Berlin, Boston: De Gruyter.

Brinker, Klaus \& Sandra Ausborn-Brinker. 2010. Linguistische Textanalyse. Eine Einführung in Grundbegriffe und Methoden (Grundlagen der Germanistik 29). Berlin: Erich Schmidt.

Schiegg, Markus. 2015. Frühmittelalterliche Glossen. Ein Beitrag zur Funktionalität und Kontextualität mittelalterlicher Schriftlichkeit (Germanistische Bibliothek 52). Heidelberg: Universitätsverlag Winter.

Schützeichel, Rudolf. 1981. Textgebundenheit. Kleinere Schriften zur mittelalterlichen deutschen Literatur. Tübingen: Max Niemeyer.

Schwarz, Alexander. 2000. Die Textsorten des Althochdeutschen. In: Werner Besch u.a. (Hg.): Sprachgeschichte. Ein Handbuch zur Geschichte der deutschen Sprache und ihrer Erforschung. 2., vollständig neu bearbeitete und erweiterte Auflage. Teilband 2 (Handbücher zur Sprach- und Kommunikationswissenschaft 2.2). Berlin, New York: De Gruyter, S. 1222-1231. 